TRANSACTIONS OF THE

AMERICAN MATHEMATICAL SOCIETY

Volume 356, Number 8 , Pages 3209-3225

S 0002-9947(03)03450-0

Article electronically published on November 25, 2003

\title{
UNCORRELATEDNESS AND ORTHOGONALITY FOR VECTOR-VALUED PROCESSES
}

\author{
PETER A. LOEB, HORST OSSWALD, YENENG SUN, AND ZHIXIANG ZHANG
}

\begin{abstract}
For a square integrable vector-valued process $f$ on the Loeb product space, it is shown that vector orthogonality is almost equivalent to componentwise scalar orthogonality. Various characterizations of almost sure uncorrelatedness for $f$ are presented. The process $f$ is also related to multilinear forms on the target Hilbert space. Finally, a general structure result for $f$ involving the biorthogonal representation for the conditional expectation of $f$ with respect to the usual product $\sigma$-algebra is presented.
\end{abstract}

\section{INTRODUCTION}

This paper considers the almost sure uncorrelatedness and almost sure orthogonality of vector-valued processes. If a vector-valued process with almost surely uncorrelated random variables is assumed to be jointly measurable in the usual sense, then the vector-valued random variables are almost surely trivial (see Remark 4.5 below). Thus a richer product measure-theoretic framework is needed to work with such processes. As demonstrated in [13], the Loeb product measure spaces do provide such a rich framework for scalar-valued processes with almost surely uncorrelated random variables. Here we show that the Loeb product framework works well in the vector case.

Our probability spaces are formed from two internal probability spaces $(T, \mathcal{A}, \nu)$ and $(\Omega, \mathcal{B}, p)$. We denote by $(T \times \Omega, \mathcal{A} \otimes \mathcal{B}, \nu \otimes p)$ the internal product space of $T$ and $\Omega$. The Loeb spaces over $(T, \mathcal{A}, \nu)$ and $(\Omega, \mathcal{B}, p)$ are denoted by $\left(T, L_{\nu}(\mathcal{A}), \widehat{\nu}\right)$ and $\left(\Omega, L_{p}(\mathcal{B}), \widehat{p}\right)$, respectively (see Chapter 5 in [1] for the detailed construction and properties of Loeb spaces). The Loeb space over the internal product $(T \times \Omega, \mathcal{A} \otimes \mathcal{B}, \nu \otimes p)$ is called the Loeb product space; it is denoted by $\left(T \times \Omega, L_{\nu \otimes p}(\mathcal{A} \otimes \mathcal{B}), \widehat{\nu \otimes p}\right)$. Although the definition of the Loeb product involves the internal factor spaces $(T, \mathcal{A}, \nu)$ and $(\Omega, \mathcal{B}, p)$, it is shown in 8] that it actually depends only on the Loeb spaces of the internal factors, i.e., only on $\left(T, L_{\nu}(\mathcal{A}), \widehat{\nu}\right)$ and $\left(\Omega, L_{p}(\mathcal{B}), \widehat{p}\right)$.

The Loeb product space is much richer than the completion of the usual product $\left(T \times \Omega, L_{\nu}(\mathcal{A}) \otimes L_{p}(\mathcal{B}), \widehat{\nu} \otimes \widehat{p}\right)$. For simplicity, we shall use the same notation

Received by the editors March 11, 2003.

2000 Mathematics Subject Classification. Primary 03H05, 28E05, 47H60; Secondary 26E35.

Key words and phrases. Vector-valued processes, Loeb product space, Keisler's Fubini Theorem, orthogonality, uncorrelatedness, multilinear functional.

The authors are grateful for the support of the National University of Singapore during the initiation of this work. 
$\left(T \times \Omega, L_{\nu}(\mathcal{A}) \otimes L_{p}(\mathcal{B}), \widehat{\nu} \otimes \widehat{p}\right)$ to denote both the usual product and its completion. As already noted in [2], $\left(T \times \Omega, L_{\nu \otimes p}(\mathcal{A} \otimes \mathcal{B}), \widehat{\nu \otimes p}\right)$ extends the product space $\left(T \times \Omega, L_{\nu}(\mathcal{A}) \otimes L_{p}(\mathcal{B}), \widehat{\nu} \otimes \widehat{p}\right)$. It is shown in [13] that the inclusion is always proper when the factor spaces $\left(T, L_{\nu}(\mathcal{A}), \widehat{\nu}\right)$ and $\left(\Omega, L_{p}(\mathcal{B}), \widehat{p}\right)$ are non-atomic (a specific example can be found in [1] p. 74). In fact, Theorem 6.2 in [13] shows that the Loeb product space is very rich in the sense that it can be endowed with independent processes that are not measurable with respect to the usual product $\sigma$-algebra $L_{\nu}(\mathcal{A}) \otimes L_{p}(\mathcal{B})$ but have almost independent random variables with any variety of distributions. In addition, it is shown in [3] that there is a continuum of increasing Loeb product null sets with large gaps in the sense that their set differences have $\widehat{\nu} \otimes \widehat{p}$-outer measure one.

The rest of this paper is organized as follows. It is obvious that the orthogonality condition for vector-valued random variables is weaker than the orthogonality condition for scalar-valued random variables obtained from each fixed component. It is surprising that when a large collection of vector-valued random variables is considered, vector orthogonality is almost equivalent to componentwise scalar orthogonality. This result is shown in Section 2. Section 3 presents a Keisler-type Fubini theorem for Banach-valued processes on Loeb product spaces. Based on this kind of Fubini property and on some standard measure-theoretic methods, we generalize in Section 4 to the vector case some results on scalar orthogonality and uncorrelatedness in [13, including the characterization of a version of the law of large numbers for vector-valued processes via almost sure uncorrelatedness. In Section 5, we relate processes taking values in separable Hilbert spaces to multilinear forms on such spaces. Many new results are obtained. A general structure result for a square integrable Hilbert space valued process on the Loeb product space is presented in Section 6 .

\section{VECTOR VERSUS COMPONENTWISE ORTHOGONALITY}

In this section we demonstrate the equivalence between the almost sure orthogonality (defined below) of an $l^{2}$-valued vector function and of its components on the Loeb product space. Note that every separable Hilbert space is isomorphic to $l^{2}$, so we can also say that this equivalence holds in general for a separable Hilbert space $\mathbb{H}$. Throughout this paper the Hilbert spaces that we work with are always assumed to be separable.

To avoid convergence problems and problems caused by the difference between the measures $\widehat{\nu \otimes p}$ and $\widehat{\nu} \otimes \widehat{p}$, we shall work with a *finite-dimensional space $\mathbb{F}$ that is closely related to $\mathbb{H}$. If $E$ is a ${ }^{*}$ finite-dimensional subspace of ${ }^{*} \mathbb{H}$ with an internal orthonormal basis $\left(\mathfrak{e}_{i}\right)_{i \leq \gamma}$, then for elements $a, b \in{ }^{*} \mathbb{H}$ we set

$$
\langle a, b\rangle_{E}:=\sum_{i \leq \gamma}\left\langle a, \mathfrak{e}_{i}\right\rangle \cdot\left\langle b, \mathfrak{e}_{i}\right\rangle=\left\langle\pi_{E}(a), \pi_{E}(b)\right\rangle,
$$

where $\pi_{E}$ denotes the orthogonal projection from ${ }^{*} \mathbb{H}$ onto $E$. Set

$$
\|a\|_{E}:=\left\|\pi_{E}(a)\right\|=\left(\sum_{i \leq \gamma}\left\langle a, \mathfrak{e}_{i}\right\rangle^{2}\right)^{\frac{1}{2}} .
$$

Note that the values $\langle a, b\rangle_{E}$ and $\|a\|_{E}$ do not depend on the choice of the orthonormal basis of $E$. If $a, b \in{ }^{*} \mathbb{H}$, then we shall write $a \approx_{E} b$ when $\|a-b\|_{E} \approx 0$. 
We will identify each $a \in \mathbb{H}$ with ${ }^{*} a \in{ }^{*} \mathbb{H}$. The following result is a straightforward consequence of saturation.

Proposition 2.1. There exists a ${ }^{*}$ finite-dimensional subspace $\mathbb{F}$ of ${ }^{*} \mathbb{H}$ such that

$(1){ }^{*} E \subset \mathbb{F}$ for each finite-dimensional subspace $E \subset \mathbb{H}$.

(2) $\left\|^{*} a\right\|_{\mathbb{F}} \approx\|a\|$ for each $a \in \mathbb{H}$.

(3) Each orthonormal basis $\left(\mathfrak{e}_{i}\right)_{i \in \mathbb{N}}$ of $\mathbb{H}$ can be extended to an internal orthonormal basis $\left(\mathfrak{e}_{i}\right)_{i \leq \gamma}$ of $\mathbb{F}$, where $\gamma$ is the internal dimension of $\mathbb{F}$.

Moreover, since the polarization identity allows the scalar product to be defined in terms of the norm, $\left\langle{ }^{*} a,{ }^{*} b\right\rangle_{\mathbb{F}} \approx\langle a, b\rangle$ for each $a, b \in \mathbb{H}$.

Fix a real number $q \geq 1$. Denote by $L^{q}(\widehat{p}, \mathbb{H})$ the set of $L_{p}(\mathcal{B})$-measurable functions $f: \Omega \rightarrow \mathbb{H}$ for which $\|f\|^{q}$ is $\widehat{p}$-integrable. We identify two functions $f$ and $g$ in $L^{q}(\widehat{p}, \mathbb{H})$ if $\|f-g\|=0 \widehat{p}$-a.s. If $\mathbb{H}=\mathbb{R}$, we will write $L^{q}(\widehat{p})$ instead of $L^{q}(\widehat{p}, \mathbb{H})$.

Let $S L^{q}(p, \mathbb{F})$ denote the set of all internal $\mathcal{B}$-measurable functions $F: \Omega \rightarrow \mathbb{F}$ such that $\omega \mapsto\|F(\omega)\|_{\mathbb{F}}^{q}$ is $S$-integrable with respect to the internal measure $p$ (see Chapter 5 in [11]). If $\mathbb{F}={ }^{*} \mathbb{R}$, we shall simply write $S L^{q}(p)$.

The following result is a slight modification of well-known results of Anderson and Loeb ([2], 9]).

Proposition 2.2. Given a function $f: \Omega \rightarrow \mathbb{H}$,

(1) $f$ is $L_{p}(\mathcal{B})$-measurable iff $f$ has a lifting $F: \Omega \rightarrow \mathbb{F}$, i.e., $F$ is internal and $\mathcal{B}$-measurable and for $\widehat{p}$-almost all $\omega \in \Omega$

$$
*(f(\omega)) \approx_{\mathbb{F}} F(\omega)
$$

(2) $f \in L^{q}(\widehat{p}, \mathbb{H})$ iff $f$ has a lifting $F: \Omega \rightarrow \mathbb{F}$ in $S L^{q}(p, \mathbb{F})$.

We call an $L_{\nu \otimes p}(\mathcal{A} \otimes \mathcal{B})$-measurable function $f: T \times \Omega \rightarrow \mathbb{H}$ an almost surely orthogonal process if $f_{s} \perp f_{t}$ for $\widehat{\nu^{2}}$-almost all $(s, t) \in T^{2}$, where $f_{s}$ and $f_{t}$ are the random variables $f(s, \cdot)$ and $f(t, \cdot)$ defined on $\Omega$, and $f_{s} \perp f_{t}$ means $\int_{\Omega}\left\langle f_{s}, f_{t}\right\rangle d \widehat{p}=$ 0.

Lemma 2.3. Fix $F: T \times \Omega \rightarrow \mathbb{F}$ in $S L^{2}(\nu \otimes p, \mathbb{F})$ and $\Phi: \Omega \rightarrow \mathbb{F}$ in $S L^{2}(p, \mathbb{F})$. Then

(1) $\left\langle F_{s}, F_{t}\right\rangle_{\mathbb{F}} \in S L^{1}(p)$ for $\widehat{\nu^{2}}$-almost all $(s, t) \in T^{2}$.

(2) $(s, t) \mapsto \int_{\Omega}\left\langle F_{s}, F_{t}\right\rangle_{\mathbb{F}} d p \in S L^{2}\left(\nu^{2}\right)$.

(3) $\left\langle F_{s}, \Phi\right\rangle_{\mathbb{F}} \in S L^{1}(p)$ for $\widehat{\nu}$-almost all $s \in T$.

(4) $s \mapsto \int_{\Omega}\left\langle F_{s}, \Phi\right\rangle_{\mathbb{F}} d p \in S L^{2}(\nu)$.

Proof. (1) By Keisler's Fubini theorem (see [7] and Chapter 5 in [1]), there exists a set $U \subseteq T$ such that $\widehat{\nu}(U)=1$ and $\left\|F_{t}\right\|_{\mathbb{F}} \in S L^{2}(p)$ for all $t \in U$. Now fix $(s, t) \in U \times U$ and an $N \in \mathcal{B}$. Then, by Hölder's inequality,

$$
\left(\int_{N}\left\langle F_{s}, F_{t}\right\rangle_{\mathbb{F}} d p\right)^{2} \leq \int_{N}\left\|F_{s}\right\|_{\mathbb{F}}^{2} d p \cdot \int_{N}\left\|F_{t}\right\|_{\mathbb{F}}^{2} d p\left\{\begin{array}{c}
\approx 0 \text { if } p(N) \approx 0 \\
\text { is limited }
\end{array}\right.
$$

This proves (1).

(2) By Keisler's Fubini Theorem, $t \mapsto \int_{\Omega}\left\|F_{t}\right\|_{\mathbb{F}}^{2} d p \in S L^{1}(\nu)$. It follows that

$$
(s, t) \mapsto \int_{\Omega}\left\|F_{s}\right\|_{\mathbb{F}}^{2} d p \cdot \int_{\Omega}\left\|F_{t}\right\|_{\mathbb{F}}^{2} d p \in S L^{1}\left(\nu^{2}\right) .
$$


Since

$$
\left(\int_{\Omega}\left\langle F_{s}, F_{t}\right\rangle_{\mathbb{F}} d p\right)^{2} \leq \int_{\Omega}\left\|F_{s}\right\|_{\mathbb{F}}^{2} d p \cdot \int_{\Omega}\left\|F_{t}\right\|_{\mathbb{F}}^{2} d p
$$

(2) is true.

The proofs of (3) and (4) are similar.

Let $\alpha$ be a real-valued random variable with mean zero and variance one on $\Omega$. Define two vector-valued random variables $\Phi$ and $\Psi$ by letting $\Phi(\omega)=(\alpha(\omega), \alpha(\omega))$ and $\Psi(\omega)=(\alpha(\omega),-\alpha(\omega))$. It is obvious that $\Phi$ and $\Psi$ are orthogonal, i.e., $\int_{\Omega}\langle\Phi, \Psi\rangle d \widehat{p}=0$; but the first component of $\Phi$ and the first component of $\Psi$ are not orthogonal. Thus vector orthogonality is not equivalent to componentwise scalar orthogonality. It is surprising that these two concepts are essentially equivalent for a large collection of vector-valued random variables (taking values in the Hilbert space $\left.l^{2}\right)$.

Theorem 2.4. Suppose $f: T \times \Omega \rightarrow l^{2}$ is in $L^{2}\left(\widehat{\nu \otimes p}, l^{2}\right)$. Then $f$ is almost surely orthogonal if and only if each component function $f^{i} \in L^{2}(\widehat{\nu \otimes p}), i=1,2, \ldots$, is almost surely orthogonal.

Proof. Since $f \in L^{2}\left(\widehat{\nu \otimes p}, l^{2}\right),\|f\| \in L^{2}(\widehat{\nu \otimes p})$. Keisler's Fubini theorem implies that there exists a $\widehat{\nu}$-full set $T_{0}$ such that $\left\|f_{t}\right\| \in L^{2}(\widehat{p})$ for all $t \in T_{0}$.

Sufficiency. Suppose for each $i, f^{i}$ is almost surely orthogonal in $L^{2}(\widehat{p})$. Then there is a $\widehat{\nu^{2}}$-full set $D_{i}$ for every $i$, such that

$$
\int_{\Omega} f_{s}^{i} f_{t}^{i} d \widehat{p}=0 \text { for all }(s, t) \in D_{i}
$$

Let $D=\left(T_{0} \times T_{0}\right) \cap\left(\bigcap_{i=1}^{\infty} D_{i}\right)$. Then $D$ is a $\widehat{\nu^{2}}$-full subset of $T \times T$. We then have that, for $(s, t) \in D, \sup _{n}\left|\sum_{i=1}^{n} f_{s}^{i}(\omega) f_{t}^{i}(\omega)\right| \leq\left\|f_{s}(\omega)\right\| \cdot\left\|f_{t}(\omega)\right\|$ for any $\omega \in \Omega$, and the Dominated Convergence Theorem implies that

$$
0=\sum_{i=1}^{\infty} \int_{\Omega} f_{s}^{i} f_{t}^{i} d \widehat{p}=\int_{\Omega} \sum_{i=1}^{\infty} f_{s}^{i} f_{t}^{i} d \widehat{p}=\int_{\Omega}\left\langle f_{s}, f_{t}\right\rangle d \widehat{p}
$$

Thus $f$ is almost surely orthogonal in $L^{2}\left(\widehat{p}, l^{2}\right)$.

Necessity. It suffices to show that for each $t^{\prime} \in T_{0}, \int_{\Omega} f_{t}^{i} f_{t^{\prime}}^{i} d \widehat{p}=0$ for $\widehat{\nu}$-almost all $t \in T$. Here, we need only show $\int_{T}\left(\int_{\Omega}\left\langle f_{t}, \varphi\right\rangle d \widehat{p}\right)^{2} d \widehat{\nu}(t)=0$ for any $\varphi \in L^{2}\left(\widehat{p}, l^{2}\right)$. We use the above propositions and lemma with $\mathbb{H}=l^{2}$. Fix an internal orthonormal basis $\left(\mathfrak{e}_{i}\right)_{i \leq \gamma}$ of $\mathbb{F}$, and fix liftings $F \in S L^{2}(\widehat{\nu \otimes p}, \mathbb{F})$ and $\Phi \in S L^{2}(\widehat{p}, \mathbb{F})$ of $f, \varphi$, respectively. Then we have

$$
\begin{gathered}
\int_{T}\left(\int_{\Omega}\left\langle f_{t}, \varphi\right\rangle d \widehat{p}\right)^{2} d \widehat{\nu}(t)=\text { (by Prop. 2.1), } \\
\left.\int_{T}\left(\int_{\Omega}{ }^{\circ}\left\langle F_{t}, \Phi\right\rangle_{\mathbb{F}} d \widehat{p}\right)^{2} d \widehat{\nu}(t)=\text { (by Lemma } 2.3(3)\right), \\
\left.\int_{T}\left(\circ \int_{\Omega}\left\langle F_{t}, \Phi\right\rangle_{\mathbb{F}} d p\right)^{2} d \widehat{\nu}(t) \approx \text { (by Lemma } 2.3(4)\right),
\end{gathered}
$$




$$
\begin{gathered}
\int_{T}\left(\int_{\Omega}\left\langle F_{t}, \Phi\right\rangle_{\mathbb{F}} d p\right)^{2} d \nu(t) \\
=\int_{T} \int_{\Omega^{2}}\left\langle F_{t}(\omega), \Phi(\omega)\right\rangle_{\mathbb{F}}\left\langle F_{t}(\lambda), \Phi(\lambda)\right\rangle_{\mathbb{F}} d p^{2}(\omega, \lambda) d \nu(t) \\
=\int_{\Omega^{2}} \sum_{i, j \leq \gamma}\left\langle\Phi(\omega), \mathfrak{e}_{i}\right\rangle\left\langle\Phi(\lambda), \mathfrak{e}_{j}\right\rangle \cdot \int_{T}\left\langle F_{t}(\omega), \mathfrak{e}_{i}\right\rangle\left\langle F_{t}(\lambda), \mathfrak{e}_{j}\right\rangle d \nu(t) d p^{2}(\omega, \lambda) \\
\leq A \cdot B \approx 0 .
\end{gathered}
$$

Here, the quantity $A$ is given by

$$
\begin{gathered}
A^{2}:=\int_{\Omega^{2}} \sum_{i, j \leq \gamma}\left\langle\Phi(\omega), \mathfrak{e}_{i}\right\rangle^{2}\left\langle\Phi(\lambda), \mathfrak{e}_{j}\right\rangle^{2} d p^{2}(\omega, \lambda) \\
=\int_{\Omega^{2}}\|\Phi(\omega)\|_{\mathbb{F}}^{2}\|\Phi(\lambda)\|_{\mathbb{F}}^{2} d p^{2}(\omega, \lambda)=\left(\int_{\Omega}\|\Phi(\omega)\|_{\mathbb{F}}^{2} d p(\omega)\right)^{2},
\end{gathered}
$$

and this is limited since $\Phi \in S L^{2}(p, \mathbb{F})$. The quantity $B$ is given by

$$
\begin{gathered}
B^{2}:=\int_{\Omega^{2}} \sum_{i, j \leq \gamma}\left(\int_{T}\left\langle F_{t}(\omega), \mathfrak{e}_{i}\right\rangle\left\langle F_{t}(\lambda), \mathfrak{e}_{j}\right\rangle d \nu(t)\right)^{2} d p^{2}(\omega, \lambda) \\
=\int_{\Omega^{2}} \sum_{i, j \leq \gamma} \int_{T^{2}}\left\langle F_{t}(\omega), \mathfrak{e}_{i}\right\rangle\left\langle F_{t}(\lambda), \mathfrak{e}_{j}\right\rangle\left\langle F_{s}(\omega), \mathfrak{e}_{i}\right\rangle\left\langle F_{s}(\lambda), \mathfrak{e}_{j}\right\rangle d \nu^{2}(t, s) d p^{2}(\omega, \lambda) \\
=\int_{T^{2}} \int_{\Omega} \sum_{i \leq \gamma}\left\langle F_{t}, \mathfrak{e}_{i}\right\rangle\left\langle F_{s}, \mathfrak{e}_{i}\right\rangle d p \cdot \int_{\Omega} \sum_{j \leq \gamma}\left\langle F_{t}, \mathfrak{e}_{j}\right\rangle\left\langle F_{s}, \mathfrak{e}_{j}\right\rangle d p d \nu^{2}(s, t) \\
=\int_{T^{2}}\left(\int_{\Omega} \sum_{i \leq \gamma}\left\langle F_{t}, \mathfrak{e}_{i}\right\rangle\left\langle F_{s}, \mathfrak{e}_{i}\right\rangle d p\right)^{2} d \nu^{2}(s, t), \\
\int_{T^{2}}\left(\int_{\Omega}\left\langle F_{t}, F_{s}\right\rangle_{\mathbb{F}} d p\right)^{2} d \nu^{2}(s, t) \approx(\text { by Lemma 2.3 }(2)), \\
\int_{T^{2}}\left(\int_{\Omega}\left\langle F_{t}, F_{s}\right\rangle_{\mathbb{F}} d p\right)^{2} d \widehat{\nu^{2}}(s, t)=(\text { by Lemma 2.3 }(1)), \\
\int_{T^{2}}\left(\int_{\Omega}\left\langle F_{t}, F_{s}\right\rangle_{\mathbb{F}} d \widehat{p}\right)^{2} d \widehat{\nu^{2}}(s, t)=\text { (by Prop. 2.1), } \\
\int_{T^{2}}\left(\int_{\Omega}\left\langle f_{t}, f_{s}\right\rangle d \widehat{p}\right)^{2} \widehat{d \nu^{2}}(s, t)=0,
\end{gathered}
$$

since $f$ is almost surely orthogonal. Thus $B$ is an infinitesimal, and hence $A \cdot B \approx 0$, and

$$
\int_{T}\left(\int_{\Omega}\left\langle f_{t}, \varphi\right\rangle d \widehat{p}\right)^{2} d \widehat{\nu}(t)=0
$$

whence we are done. 


\section{Vector-VAlued Fubini theOrem on Loeb Product SPACE}

In this section we extend Keisler's Fubini theorem to the Banach case, even though only the Hilbert-valued case is needed here in later sections. We will need the following well-known result.

Lemma 3.1. Let $\Psi$ be a bounded linear operator from a Banach space $X$ to a Banach space $Y$. If $f$ is Bochner integrable with respect to measure $\mu$ on a measurable space $(S, \mathcal{S})$, then for each $U \in \mathcal{S}$,

$$
\Psi\left(\int_{U} f d \mu\right)=\int_{U} \Psi f d \mu .
$$

Proof. See, e.g., [15] or [6].

Theorem 3.2 (Keisler's Fubini theorem for vector-valued random variables). Fix $f: T \times \Omega \rightarrow \mathbb{B}$ in $L^{1}(\widehat{\nu \otimes p}, \mathbb{B})$, where $(\mathbb{B},\|\cdot\|)$ is a Banach space. Then

(1) For $\widehat{\nu}$-almost all $t \in T, f(t, \cdot)$ is Bochner integrable with respect to $\widehat{p}$;

(2) $t \mapsto \int_{\Omega} f_{t} d \widehat{p}$ is Bochner integrable with respect to $\widehat{\nu}$; and

(3) $\int_{T \times \Omega} f d \widehat{\nu \otimes p}=\int_{T} \int_{\Omega} f_{t} d \widehat{p} d \widehat{\nu}(t)$.

Proof. Since the function $f$ is Bochner integrable, there exists a sequence $\left(f_{n}\right)_{n \in \mathbb{N}}$ of $L_{\nu \otimes p}(\mathcal{A} \otimes \mathcal{B})$-measurable simple functions such that

(i) $\left(\left\|f_{n}-f\right\|\right)_{n \in \mathbb{N}}$ is a decreasing sequence of non-negative functions and tends to $0 \widehat{\nu \otimes p}$-a.s., and

(ii) $\lim _{n \rightarrow \infty} \int_{T \times \Omega}\left\|f_{n}-f\right\| d \widehat{\nu \otimes p}=0$.

(See G. Da Prato, J. Zabczyk [5].)

It follows, by Keisler's scalar-valued Fubini theorem and Lebesgue's theorem, that there exists a $\widehat{\nu}$-full set $T_{1}$ such that for all $t \in T_{1}$,

(iii) $\lim _{n \rightarrow \infty}\left\|f_{n}(t, \cdot)-f(t, \cdot)\right\|=0 \widehat{p}$-a.s.,

(iv) $\lim _{n \rightarrow \infty} \int_{\Omega}\left\|f_{n}(t, \cdot)-f(t, \cdot)\right\| d \widehat{p}=0$.

It follows that $f_{t}$ is Bochner integrable for all $t \in T_{1}$, whence (1) is true.

Fix $t \in T_{1}$. It follows from (iv) and the fact that $\int_{\Omega} f_{n}(t, \cdot) d \widehat{p}$ is $L_{\nu}(\mathcal{A})$ measurable that $\int_{\Omega} f_{t} d \widehat{p}$ is $L_{\nu}(\mathcal{A})$-measurable. Since

$$
\int_{T}\left\|\int_{\Omega} f_{t} d \widehat{p}\right\| d \widehat{\nu}(t) \leq \int_{T \times \Omega}\|f\| d \widehat{\nu \otimes p}<\infty,
$$

$t \mapsto \int_{\Omega} f_{t} d \widehat{p}$ is $\widehat{\nu}$-Bochner integrable. Thus (2) is true.

Now we turn to the proof of (3). It follows from Lemma 3.1 and the scalar-valued Keisler Fubini theorem that for every $\varphi \in \mathbb{B}^{\prime}$ (the topological dual space of $\mathbb{B}$ )

$$
\varphi\left(\int_{T \times \Omega} f d \widehat{\nu \otimes p}\right)=\varphi\left(\int_{T} \int_{\Omega} f_{t} d \widehat{p} d \widehat{\nu}(t)\right) .
$$

Thus $\int_{T \times \Omega} f d \widehat{\nu \otimes p}=\int_{T} \int_{\Omega} f_{t} d \widehat{p} d \widehat{\nu}(t)$. This finishes the proof.

\section{Properties of Vector uncorrelatedness And orthogonality}

Let $\mathcal{U}$ denote the conventional product $L_{\nu}(\mathcal{A}) \otimes L_{p}(\mathcal{B})$ of respective Loeb spaces. In this section it will be shown that the almost sure uncorrelatedness and orthogonality for Hilbert space valued vectors on the Loeb product space can be characterized by the conditional expectation with respect to $\mathcal{U}$. For a $\widehat{p}$-integrable function $g$ on $\Omega$, denote the integral $\int_{\Omega} g d \widehat{p}$ by $E g$. 
We call an $L_{\nu \otimes p}(\mathcal{A} \otimes \mathcal{B})$-measurable function $f: T \times \Omega \rightarrow \mathbb{H}$ almost surely uncorrelated if $\mathrm{E}\left\langle f_{s}, f_{t}\right\rangle=\left\langle\mathrm{E} f_{s}, \mathrm{E} f_{t}\right\rangle$ for $\widehat{\nu^{2}}$-almost all $(s, t) \in T^{2}$. For each $\omega \in \Omega$, the sample function $f(\cdot, \omega)$ defined on $T$ is denoted by $f_{\omega}$. We have the following results resembling those in the scalar-value case in [13] and [14].

Lemma 4.1. Fix a function $f: T \times \Omega \rightarrow \mathbb{H}$ in $L^{1}(\widehat{\nu \otimes p}, \mathbb{H})$. If $E(f \mid \mathcal{U})=h$, where $h$ is an element in $L^{1}\left(\left(T, L_{\nu}(\mathcal{A}), \widehat{\nu}\right), \mathbb{H}\right)$, then for $\widehat{p}$-almost all $\omega \in \Omega, f_{\omega}$ is integrable on $\left(T, L_{\nu}(\mathcal{A}), \widehat{\nu}\right)$, and for any given $A \in L_{\nu}(\mathcal{A}), \int_{A} f_{\omega} d \widehat{\nu}=\int_{A} h_{t} d \widehat{\nu}(t)$ for $\widehat{p}$-almost all $\omega \in \Omega$. Furthermore, $h_{t}=E f_{t}$ for $\widehat{\nu}$-almost all $t \in T$.

Proof. By Theorem 3.2, $\mathrm{E} f_{t}$ is $L_{\nu}(\mathcal{A})$-measurable. Theorem 3.2 also yields the result that for each $A \in L_{\nu}(\mathcal{A}), B \in L_{\nu}(\mathcal{B})$,

$$
\begin{aligned}
& \int_{B} \int_{A} f(t, \omega) d \widehat{\nu}(t) d \widehat{p}(\omega)=\int_{A \times B} f d \widehat{\nu \otimes p}=\int_{A \times B} \mathrm{E}(f \mid \mathcal{U}) d \widehat{\nu \otimes p} \\
= & \int_{A \times B} h d \widehat{\nu \otimes p}=\int_{B} \int_{A} h d \widehat{\nu} d \widehat{p} .
\end{aligned}
$$

Note that $\mathbb{H}$ is a separable Hilbert space. Choose a sequence $\left\{\varphi_{n}\right\}_{n=1}^{\infty}$ that is dense in $\mathbb{H}^{\prime}$. Then for any fixed $n \geq 1$ and $A \in L_{\nu}(\mathcal{A})$,

$$
\int_{B} \varphi_{n}\left(\int_{A} f(t, \omega) d \widehat{\nu}(t)\right) d \widehat{p}(\omega)=\int_{B} \varphi_{n}\left(\int_{A} h d \widehat{\nu}\right) d \widehat{p}
$$

holds for all $B \in L_{\nu}(\mathcal{B})$. By the uniqueness of the Radon-Nikodým derivatives and by grouping countably many null sets together, it follows that for $\widehat{p}$-almost all $\omega \in \Omega$, $\varphi_{n}\left(\int_{A} f_{\omega} d \widehat{\nu}\right)=\varphi_{n}\left(\int_{A} h_{t} d \widehat{\nu}(t)\right)$ holds for all $n \geq 1$. Thus, $\int_{A} f_{\omega} d \widehat{\nu}=\int_{A} h_{t} d \widehat{\nu}(t)$.

Note that we also have

$$
\int_{A} \int_{\Omega} f d \widehat{p} d \widehat{\nu}=\int_{A} \int_{\Omega} h d \widehat{p} d \widehat{\nu}=\int_{A} h d \widehat{\nu}
$$

for any $A \in L_{\nu}(\mathcal{A})$. By the same argument as above, we have $h_{t}=\mathrm{E} f_{t}$ for $\widehat{\nu}$-almost all $t \in T$.

As in the scalar case in [13, 14, we say that the vector valued process $f$ satisfies the consistency law if the first result in the above lemma holds, that is, if for any given $A \in L_{\nu}(\mathcal{A}), \int_{A} f_{\omega} d \widehat{\nu}=\int_{A} h_{t} d \widehat{\nu}(t)$ for $\widehat{p}$-almost all $\omega \in \Omega$, where $h$ is an element in $L^{1}\left(\left(T, L_{\nu}(\mathcal{A}), \widehat{\nu}\right), \mathbb{H}\right)$.

To characterize the almost sure orthogonality, we say the random variables $f_{t}$ are almost surely orthogonal as defined in Section 2, i.e., $\mathrm{E}\left\langle f_{s}, f_{t}\right\rangle=0$ for $\widehat{\nu^{2}}$-almost all $(s, t) \in T^{2}$. The sample functions $f_{\omega}$ are said to be almost surely orthogonal if $\int_{T}\left\langle f_{\omega_{1}}(t), f_{\omega_{2}}(t)\right\rangle d \widehat{\nu}(t)=0$ for $\widehat{p^{2}}$-almost all $\left(\omega_{1}, \omega_{2}\right) \in \Omega^{2}$. We will show these two definitions are equivalent. For this purpose we first prove the following properties about orthogonality.

Lemma 4.2. Fix $f: T \times \Omega \rightarrow \mathbb{H} \in L^{2}(\widehat{\nu \otimes p}, \mathbb{H})$.

(i) If the random variables $f_{t}$ are almost surely orthogonal in $L^{2}(\widehat{p}, \mathbb{H})$, then $E(f \mid \mathcal{U})=0$, and for all $A \in L_{\nu}(\mathcal{A}), \int_{A} f_{\omega} d \widehat{\nu}=0$ for $\widehat{p}$-almost all $\omega \in \Omega$.

(ii) If the sample functions $f_{\omega}$ are almost surely orthogonal in $L^{2}(\widehat{\nu}, \mathbb{H})$, then $E(f \mid \mathcal{U})=0$, and for all $B \in L_{\nu}(\mathcal{B}), \int_{B} f(t, \omega) d \widehat{p}(\omega)=0$ for $\widehat{\nu}$-almost all $t \in T$, in particular, $E f_{t}=0$ for $\widehat{\nu}$-almost all $t \in T$. 
Proof. Suppose $f_{t}$ 's are almost surely orthogonal. Keisler's Fubini theorem implies that for all $A \in L_{\nu}(\mathcal{A})$,

$$
\begin{aligned}
0 & =\int_{A \times A} \mathrm{E}\left\langle f_{t_{1}}, f_{t_{2}}\right\rangle \widehat{d \nu^{2}}\left(t_{1}, t_{2}\right) \\
& =\int_{\Omega}\left\langle\int_{A} f_{t_{1}} d \widehat{\nu}\left(t_{1}\right), \int_{A} f_{t_{2}} d \widehat{\nu}\left(t_{2}\right)\right\rangle d \widehat{p}=\mathrm{E}\left(\left\|\int_{A} f_{t} d \widehat{\nu}(t)\right\|\right)^{2} .
\end{aligned}
$$

Thus $\int_{A} f_{t} d \widehat{\nu}(t)=0, \widehat{p}$-a.s. It follows from Keisler's Fubini theorem that the integral $\int_{A \times B} f d \widehat{\nu \otimes p}=0$ for any $A \in L_{\nu}(\mathcal{A}), B \in L_{p}(\mathcal{B})$. Since $\left\{D \in \mathcal{U}: \int_{D} f d \widehat{\nu \otimes p}=0\right\}$ is a sigma-field containing all $A_{1} \times B_{1}$ with $A_{1} \in L_{\nu}(\mathcal{A}), B_{1} \in L_{p}(\mathcal{B})$, the Monotone Class Theorem (see, e.g., [4]), implies that $\int_{D} f d \widehat{\nu \otimes p}=0$ for all $D \in \mathcal{U}$. Thus $\mathrm{E}(f \mid \mathcal{U})=0$.

If the $f_{\omega}$ 's are almost surely orthogonal, the corresponding results can be proved similarly by symmetry.

Theorem 4.3. For $f: T \times \Omega \rightarrow \mathbb{H}$ in $L^{2}(\widehat{\nu \otimes p}, \mathbb{H})$, the following statements are equivalent:

(1) $E(f \mid \mathcal{U})=0$;

(2) the $f_{t}$ 's are almost surely orthogonal in $L^{2}(\widehat{p}, \mathbb{H})$;

(3) the $f_{\omega}$ 's are almost surely orthogonal in $L^{2}(\widehat{\nu}, \mathbb{H})$.

Proof. $(1) \Longrightarrow(2)$ : Suppose $\mathrm{E}(f \mid \mathcal{U})=0$. It follows from Lemma 4.1 that $\mathrm{E} f_{t}=0$ for $\widehat{\nu}$-almost all $t$. Since $f \in L^{2}(\widehat{\nu \otimes p}, \mathbb{H})$, Keisler's Fubini Theorem implies that there is a $\widehat{\nu}$-full set $T_{0}$ such that for all $t \in T_{0}, f_{t} \in L^{2}(\widehat{p}, \mathbb{H})$. Therefore for $t^{\prime} \in T_{0}$, $\mathrm{E}\left[\left\langle f, f_{t^{\prime}}\right\rangle \mid \mathcal{U}\right](t, \omega)=0$. By Lemma 4.1, $\int_{\Omega}\left\langle f_{t}, f_{t^{\prime}}\right\rangle d \widehat{p}=0$ for $\widehat{\nu}$-almost all $t \in T$. Keisler's Fubini theorem implies that for $\widehat{\nu^{2}}$-almost all $\left(t, t^{\prime}\right), \mathrm{E}\left[\left\langle f_{t}, f_{t^{\prime}}\right\rangle\right]=0$, i.e., the $f_{t}$ 's are almost surely orthogonal in $L^{2}(\widehat{p}, \mathbb{H})$.

By symmetry, $(1) \Longrightarrow(3)$ follows.

$(2) \Longrightarrow(1)$ and $(3) \Longrightarrow(1)$ have been proved in Lemma 4.2

Next we characterize uncorrelatedness.

Theorem 4.4. Fix $f: T \times \Omega \rightarrow \mathbb{H}$ in $L^{1}(\widehat{\nu \otimes p}, \mathbb{H})$. The following are equivalent:

(1) $E(f \mid \mathcal{U})=h$, where $h$ is an integrable function on $\left(T, L_{\nu}(\mathcal{A}), \widehat{\nu}\right)$.

(2) $f$ satisfies the consistency law, i.e., for any given $A \in L_{\nu}(\mathcal{A}), \int_{A} f_{\omega} d \widehat{\nu}=$ $\int_{A \times \Omega} f d \widehat{\nu \otimes p}$ for $\widehat{p}$-almost all $\omega \in \Omega$.

Furthermore, for $f: T \times \Omega \rightarrow \mathbb{H}$ in $L^{2}(\widehat{\nu \otimes p}, \mathbb{H})$, both (1) and (2) are equivalent to either of the following:

(3) The $f_{t}$ 's are almost surely uncorrelated.

(4) The centered random variables $f_{t}-E f_{t}$ are almost surely orthogonal.

Proof. (1) $\Longrightarrow(2)$ : This follows from Lemma 4.1

$(2) \Longrightarrow(1)$ : For all $A \in L_{\nu}(\mathcal{A}), \int_{A} f_{\omega} d \widehat{\nu}=\int_{A} \mathrm{E} f_{t} d \widehat{\nu}(t)$ for $\widehat{p}$-almost all $\omega \in \Omega$. Thus for any $A \in L_{\nu}(\mathcal{A})$ and $B \in L_{p}(\mathcal{B})$,

$$
\begin{aligned}
\int_{A \times B} f d \widehat{p \otimes \nu} & =\int_{B} \int_{A} f_{\omega} d \widehat{\nu} d \widehat{p}(\omega) \\
& =\int_{B} \int_{A} \mathrm{E} f_{t} d \widehat{\nu}(t) d \widehat{p}=\int_{A \times B} \mathrm{E} f_{t} d \widehat{\nu \otimes p}(t, \cdot)
\end{aligned}
$$


Thus we obtain (1) by the similar argument as in the proof of Lemma 4.2 using the Monotone Class Theorem.

$(3) \Longleftrightarrow(4)$ : This follows from the identity that

$$
\mathrm{E}\left[\left\langle f_{t}-\mathrm{E} f_{t}, f_{s}-\mathrm{E} f_{s}\right\rangle\right]=\mathrm{E}\left[\left\langle f_{t}, f_{s}\right\rangle\right]-\left\langle\mathrm{E} f_{t}, \mathrm{E} f_{s}\right\rangle
$$

for all $t, s \in T$.

$(4) \Longrightarrow(1)$ : This follows from Lemma 4.2

$(1) \Longrightarrow(4)$ : It follows from Lemma 4.1 that $h_{t}=\mathrm{E} f_{t}$ for $\widehat{\nu}$-almost all $t$. Let $e(t, \omega)=f(t, \omega)-\mathrm{E} f_{t}$. Then $\mathrm{E}(e \mid \mathcal{U})=0$. By Theorem 4.3, $e$ is an almost surely orthogonal process; i.e., the centered random variables $f_{t}-\mathrm{E} f_{t}$ are almost surely orthogonal.

Remark 4.5. We know from Theorem 4.4 that if an almost surely uncorrelated Hilbert-valued process $f$ is measurable with respect to the usual product $\sigma$-algebra $\mathcal{U}=L_{\nu}(\mathcal{A}) \otimes L_{p}(\mathcal{B})$, then for $\widehat{\nu}$-almost all $t \in T$, the random variable $f_{t}(\cdot)$ is nonrandom. Note that this type of result holds not only for the product of two Loeb spaces but also for the product of any two probability spaces as in Proposition 1.1 of [14. We mention here that it is shown in Theorem 6.2 of 13 that there are many almost surely pairwise independent processes on the Loeb product spaces. Therefore there are many almost surely uncorrelated processes; see Remark 5.9 below.

\section{Applications to multilinear forms}

In this section we apply the results above to continuous multilinear forms. Throughout the section, $\mathbb{H}, \mathbb{H}_{i}, \ldots, \mathbb{H}_{n}$ are separable Hilbert spaces. For brevity, their norms are simply expressed by $\|\cdot\|$. There should be no confusion.

Multilinear forms play an important role in Malliavin Calculus: let $C_{\mathbb{B}}$ be the space of continuous functions, defined on $[0,1]$, with values in an abstract Wiener space $\mathbb{B}$ over $\mathbb{H}$ : let $W$ be the Wiener measure on $C_{\mathbb{B}}$. Then the kernel of the $n$-th level of the chaos decomposition of a functional $\varphi \in L^{2}(W)$ is a function $g$, defined on $[0,1]^{n}$, with values in the space of continuous $n$-fold real multilinear forms on $\mathbb{H}$. Thus $g: \mathbb{H}_{1} \times \cdots \times \mathbb{H}_{n} \times[0,1]^{n} \rightarrow \mathbb{R}$ with $\mathbb{H}_{i}=\mathbb{H}$. Since the Lebesgue space $[0,1]^{n}$ can be represented by a Loeb probability space $T^{n}$, we may assume that $g: \mathbb{H}_{1} \times \cdots \times \mathbb{H}_{n} \times T^{n} \rightarrow \mathbb{R}$. For more details see [12].

Note that, since $\mathbb{H}=\mathbb{H}^{\prime}$, the dual of $\mathbb{H}$, a multilinear function on $\mathbb{H}_{1} \times \cdots \times \mathbb{H}_{n}$ with values in $\mathbb{H}$ is a real-valued multilinear function, defined on $\mathbb{H}_{1} \times \cdots \times \mathbb{H}_{n} \times \mathbb{H}$. Therefore it suffices to study real-valued continuous multilinear functions (called multilinear forms).

The following lemma provides a tool to verify the integrability. Its routine proof is omitted.

Lemma 5.1. If $\Psi: \mathbb{H}_{1} \times \cdots \times \mathbb{H}_{n} \rightarrow \mathbb{R}$ is multilinear (that is, $\Psi\left(h_{1}, \ldots, h_{i}, \ldots, h_{n}\right)$ is linear in each $h_{i}$ ) and continuous, then there exists a constant $A>0$ such that for any $h_{i} \in \mathbb{H}_{i}, i=1, \ldots, n$,

$$
\left|\Psi\left(h_{1}, \ldots, h_{n}\right)\right| \leq A \prod_{i=1}^{n}\left\|h_{i}\right\| .
$$

The following result is crucial in proving other results on multilinear forms in this section. 
Theorem 5.2. Let $\Phi: \mathbb{H}_{1} \times \cdots \times \mathbb{H}_{n} \times \Omega \rightarrow \mathbb{R}$ be a Borel $\otimes$ Loeb measurable function, and $f^{i}$ an $\mathbb{H}_{i}$-valued process on $T \times \Omega$ for each $i \in\{1, \ldots, n\}$. Suppose that for some $j, \Phi\left(h_{1}, \ldots, h_{j}, \ldots, h_{n}, \omega\right)$ is linear and continuous with respect to $h_{j}$, and $f^{j}$ is an almost surely orthogonal process. Assume that $\Phi\left(f^{1}\left(t_{1}, \omega\right), \ldots, f^{n}\left(t_{n}, \omega\right), \omega\right)$ is integrable on $\left(T^{n} \times \Omega, L_{\nu^{n}} \otimes p\left(\mathcal{A}^{n} \otimes \mathcal{B}\right), \widehat{\nu^{n} \otimes p}\right)$. Then it follows that for $\widehat{\nu^{n}}$-almost all $\left(t_{1}, \ldots, t_{n}\right) \in T^{n}$,

$$
\int_{\Omega} \Phi\left(f_{t_{1}}^{1}(\omega), \ldots, f_{t_{n}}^{n}(\omega), \omega\right) d \widehat{p}(\omega)=0 .
$$

Proof. Without loss of generality, suppose $j=1$. By Keisler's Fubini theorem in Section 3 , there is a $\widehat{\nu^{n-1}}$-full set $D \subset T^{n-1}$, such that for any $\left(t_{2}, \ldots, t_{n}\right) \in D$, $g(t, \omega):=\Phi\left(f_{t}^{1}(\omega), f_{t_{2}}^{2}(\omega), \ldots, f_{t_{n}}^{n}(\omega), \omega\right) \in L^{1}(\widehat{\nu \otimes p}, \mathbb{H})$. It suffices to show that $\mathrm{E} g_{t}=0$ for $\widehat{\nu}$-almost all $t \in T$. For this, it suffices to show that $\mathrm{E}[g \mid \mathcal{U}]=0$ by Theorem 4.3 By Lemmas 3.1 and 4.1, and Theorem 3.2. we have for all $A \in$ $L_{\nu}(\mathcal{A}), B \in L_{p}(\mathcal{B})$,

$$
\begin{aligned}
& \int_{A \times B} g d \widehat{\nu \otimes p} \\
= & \int_{A \times B} \Phi\left(f^{1}(t, \omega), f_{t_{2}}^{2}(\omega), \ldots, f_{t_{n}}^{n}(\omega), \omega\right) d \widehat{\nu \otimes p}(t, \omega) \\
= & \int_{B} \int_{A} \Phi\left(f^{1}(t, \omega), f_{t_{2}}^{2}(\omega), \ldots, f_{t_{n}}^{n}(\omega), \omega\right) d \widehat{\nu}(t) d \widehat{p}(\omega) \\
= & \int_{B} \Phi\left(\int_{A} f^{1}(t, \omega) d \widehat{\nu}(t), f_{t_{2}}^{2}(\omega), \ldots, f_{t_{n}}^{n}(\omega), \omega\right) d \widehat{p}(\omega) \\
= & \int_{B} \Phi\left(0, f_{t_{2}}^{2}(\omega), \ldots, f_{t_{n}}^{n}(\omega), \omega\right) d \widehat{p}(\omega)=0,
\end{aligned}
$$

whence we are done.

Remark 5.3. If $\Phi\left(f_{t}^{1}(\omega), f_{t_{2}}^{2}(\omega), \ldots, f_{t_{n}}^{n}(\omega), \omega\right) \in L^{2}\left(\widehat{\nu^{n} \otimes p}, \mathbb{H}\right)$, then Theorem 4.3 implies that the process $g(t, \omega)$ is almost surely orthogonal.

It should also be mentioned that there exists a Loeb space $(\Omega, \widehat{p})$ that represents the probability space $\left(C_{\mathbb{B}}, W\right)$. Here, the Malliavin derivative of a Malliavin differentiable functional $\varphi \in L^{2}(\widehat{p})$ belongs to $L^{2}(\widehat{\nu \otimes p}, \mathbb{H})$ (see [12]).

Corollary 5.4. For $i=1, \ldots, n$, fix $f^{i}: T \times \Omega \rightarrow \mathbb{H}_{i}$ and $\varphi^{i}: \Omega \rightarrow \mathbb{H}_{i}$. Suppose that (1) for each $i, f^{i} \in L^{2 n}\left(\widehat{\nu \otimes p}, \mathbb{H}_{i}\right)$ and $\varphi^{i} \in L^{2 n}\left(\widehat{p}, \mathbb{H}_{i}\right)$; or (2) for each $i$, $\varphi^{i} \in \mathbb{H}_{i}$ is deterministic and $f^{i} \in L^{n}\left(\widehat{\nu \otimes p}, \mathbb{H}_{i}\right)$. Assume that one of the processes $f^{i}$ is almost surely orthogonal. Then for $\widehat{\nu^{n}}$-almost all $\left(t_{1}, \ldots, t_{n}\right) \in T^{n}$,

$$
E \prod_{i=1}^{n}\left\langle f_{t_{i}}^{i}, \varphi^{i}\right\rangle=0 .
$$

Proof. This follows from Theorem 5.2 by taking $\Phi\left(h_{1}, \ldots, h_{n}, \omega\right)=\prod_{i=1}^{n}\left\langle h_{i}, \varphi^{i}(\omega)\right\rangle$. Note that $\prod_{i=1}^{n}\left\langle f^{i}, \varphi^{i}\right\rangle \in L^{1}\left(\widehat{\nu^{n} \otimes p}, \mathbb{R}\right)$ follows from Hölder's inequality.

By symmetry, we also have results similar to those in Theorem 5.2 for a $t$ parameterized function that is continuous and linear with respect to some of its arguments. Here is an example. 
Proposition 5.5. Suppose $f: T \times \Omega \rightarrow \mathbb{H}$ in $L^{2}(\widehat{\nu \otimes p}, \mathbb{H})$ is almost surely orthogonal, and fix a bounded measurable function $h: T \rightarrow \mathbb{H}$ and $\Psi$, a bilinear form on $\mathbb{H} \times \mathbb{H}$. Then the process $g$ defined by $g(t, \omega)=\Psi(f(t, \omega), h(t))$ is also almost surely orthogonal.

Proof. Note that $g$ is still square integrable. For all $A \in L_{\nu}(\mathcal{A}), B \in L_{\nu}(\mathcal{B})$, Lemma 4.2 implies that

$$
\int_{A \times B} \Psi(f, h) d \widehat{\nu \otimes p}=\int_{A} \Psi\left(\int_{B} f d \widehat{p}, h\right) d \widehat{\nu}=\int_{A} \Psi(0, h) d \widehat{\nu}=0 .
$$

Thus, $\mathrm{E}[g \mid \mathcal{U}]=0$. The result then follows from Theorem 4.3,

The following theorem relates almost surely uncorrelated processes with stochastic multilinear forms.

Theorem 5.6. Let $n \geq 2$ be a fixed integer. Suppose that for all $i \in\{1, \ldots, n\}$, $f^{i}: T \times \Omega \rightarrow \mathbb{H}_{i}$ in $L^{n}\left(\widehat{\nu \otimes p}, \mathbb{H}_{i}\right)$ are almost surely uncorrelated processes. Let $\Phi: \mathbb{H}_{1} \times \cdots \times \mathbb{H}_{n} \times \Omega \rightarrow \mathbb{R}$ be a measurable function, continuous and multilinear with respect to arguments in $\mathbb{H}_{1} \times \cdots \times \mathbb{H}_{n}$ that satisfies the following condition for a positive constant $A$ :

$$
\left|\Phi\left(h_{1}, \ldots, h_{n}, \omega\right)\right| \leq A \prod_{i=1}^{n}\left\|h_{i}\right\|, h_{i} \in \mathbb{H}_{i}, i=1, \ldots, n, \omega \in \Omega .
$$

Then for $\widehat{\nu^{n}}$-almost all $\left(t_{1}, \ldots, t_{n}\right) \in T^{n}$,

$$
\int_{\Omega} \Phi\left(f_{t_{1}}^{1}, \ldots, f_{t_{n}}^{n}, \omega\right) d \widehat{p}(\omega)=\int_{\Omega} \Phi\left(\int_{\Omega} f_{t_{1}}^{1} d \widehat{p}, \ldots, \int_{\Omega} f_{t_{n}}^{n} d \widehat{p}, \omega\right) d \widehat{p}(\omega) .
$$

Proof. Note that the processes $f_{t_{i}}^{i}-\mathrm{E} f_{t_{i}}^{i}, i=1, \ldots, n$, are almost surely orthogonal. Repeatedly applying Theorem [5.2, we almost surely have

$$
\begin{aligned}
& \mathrm{E}\left[\Phi\left(f_{t_{1}}^{1}, \ldots, f_{t_{n}}^{n}, \cdot\right)\right] \\
= & \mathrm{E} \Phi\left(f_{t_{1}}^{1}-\mathrm{E} f_{t_{1}}^{1}, f_{t_{2}}^{2} \ldots, f_{t_{n}}^{n}, \cdot\right)+\mathrm{E} \Phi\left(\mathrm{E} f_{t_{1}}^{1}, f_{t_{2}}^{2} \ldots, f_{t_{n}}^{n}, \cdot\right) \\
= & \mathrm{E} \Phi\left(\mathrm{E} f_{t_{1}}^{1}, f_{t_{2}}^{2} \ldots, f_{t_{n}}^{n}, \cdot\right) \\
= & \ldots \ldots \\
= & \mathrm{E} \Phi\left(\mathrm{E} f_{t_{1}}^{1}, \ldots, \mathrm{E} f_{t_{n-1}}^{n-1}, \mathrm{E} f_{t_{n}}^{n}, \cdot\right) .
\end{aligned}
$$

Note that all the integrals exist by Hölder's inequality.

Recall that membership of $\varphi: \Omega \rightarrow \mathbb{H}$ in $L^{\infty}(\widehat{p}, \mathbb{H})$ means that $\|\varphi\|: \Omega \rightarrow \mathbb{R}$ is in $L^{\infty}(\widehat{p})$.

Theorem 5.7. Fix $f^{i}: T \times \Omega \rightarrow \mathbb{H}_{i}$ in $L^{n}\left(\widehat{\nu \otimes p}, \mathbb{H}_{i}\right)$ and $\varphi^{i}: \Omega \rightarrow \mathbb{H}_{i}$ in $L^{\infty}\left(\widehat{p}, \mathbb{H}_{i}\right), i=1, \ldots, n$, where $n$ is a positive integer. Suppose that $f^{i}$ is almost surely uncorrelated for $i=1, \ldots, n$. Suppose $\varphi^{1}, \ldots, \varphi^{n}$ are mutually independent. Then for $\widehat{\nu^{n}}$-almost all $\left(t_{1}, \ldots, t_{n}\right)$ in $T^{n}$,

$$
E\left[\prod_{i}\left\langle f_{t_{i}}^{i}, \varphi^{i}\right\rangle\right]=\prod_{i}\left\langle E f_{t_{i}}^{i}, E \varphi^{i}\right\rangle .
$$


Proof. Note that for $h_{i} \in \mathbb{H}_{i}, i=1, \ldots, n, \Psi\left(h_{1}, \ldots, h_{n}, \omega\right):=\prod_{i}\left\langle h_{i}, \varphi^{i}(\omega)\right\rangle$ is continuous and multilinear with respect to $\left(h_{1}, \ldots, h_{n}\right)$, thus satisfying all the conditions in Theorem [5.6 with $A$ being the product of the $\widehat{p}$-essential bounds of $\left\|\varphi^{i}(\omega)\right\|$. Thus $\mathrm{E}\left[\prod_{i}\left\langle f_{t_{i}}^{i}, \varphi^{i}\right\rangle\right]=\mathrm{E} \prod_{i}\left\langle\mathrm{E} f_{t_{i}}^{i}, \varphi^{i}\right\rangle$ for $\widehat{\nu n}^{n}$ almost all $\left(t_{1}, \ldots, t_{n}\right) \in$ $T^{n}$. By the mutual independence of the $\varphi^{i}$ s,, $\mathrm{E} \prod_{i}\left\langle\mathrm{E} f_{t_{i}}^{i}, \varphi^{i}\right\rangle=\prod_{i} \mathrm{E}\left\langle\mathrm{E} f_{t_{i}}^{i}, \varphi^{i}\right\rangle$. The result then follows from Lemma 3.1.

The next theorem extends Theorem 2.4.

Theorem 5.8. Fix $f: T \times \Omega \rightarrow \mathbb{H}$ in $L^{2}(\widehat{\nu \otimes p}, \mathbb{H})$. Then $f$ is almost surely orthogonal (resp., uncorrelated) if and only if for every $a \in \mathbb{H}$, the real-valued process $\left\langle f_{t}, a\right\rangle$ is almost surely orthogonal ( resp., uncorrelated).

Proof. Note that for $a \in \mathbb{H},\langle f, a\rangle \in L^{2}(\widehat{\nu \otimes p})$. By Theorem 4.4, we need only prove the orthogonality result.

The proof of sufficiency is similar to the proof of the sufficiency in Theorem 2.4 by replacing the $i$ th component $f^{i}$ by $\left\langle f, a_{i}\right\rangle$, where $\left\{a_{1}, a_{2}, \ldots\right\}$ is an orthonormal basis of $\mathbb{H}$.

Necessity follows from Theorem 5.7 and Lemma 4.2 by taking $n=2, \mathbb{H}_{1}=\mathbb{H}_{2}=$ $\mathbb{H}, f^{1}=f^{2}=f$ (note that here $f^{i}$ is not the $i$ th component of $f$ ), and $\varphi^{1}=\varphi^{2} \equiv a$ (non random).

Remark 5.9. Fix $f: T \times \Omega \rightarrow \mathbb{H} \in L^{2}(\widehat{\nu \otimes p}, \mathbb{H})$. Suppose that $f$ is almost surely pairwise independent. Then for any $a \in \mathbb{H}$, the process $\langle a, f\rangle$ is still almost surely pairwise independent, and thus almost surely uncorrelated. Hence Theorem 5.8 implies that the process $f$ is almost surely uncorrelated.

The following is a corollary of Theorem 5.6

Corollary 5.10. Let $n \geq 2$ be a fixed integer. Suppose that for all $i \in\{1, \ldots, n\}$, $f^{i}: T \times \Omega \rightarrow \mathbb{H}_{i}$ is in $L^{n}\left(\widehat{\nu \otimes p}, \mathbb{H}_{i}\right)$. Suppose for each fixed $i, f_{\omega}^{i}, \omega \in \Omega$, are almost surely exchangeable, i.e., there is a distribution $\mu_{i}$ on $\mathbb{H}_{i} \times \mathbb{H}_{i}$ such that for $\widehat{p^{2}}$-almost all $\left(\omega_{1}, \omega_{2}\right),\left(f_{\omega_{1}}^{i}, f_{\omega_{2}}^{i}\right)$ has distribution $\mu_{i}$. Let $\Phi: \mathbb{H}_{1} \times \cdots \times \mathbb{H}_{n} \rightarrow \mathbb{R}$ be a multilinear form on $\mathbb{H}_{1} \times \cdots \times \mathbb{H}_{n}$. We then have for $\widehat{\nu^{n}}$-almost all $\left(t_{1}, \ldots, t_{n}\right)$,

$$
\int_{\Omega} \Phi\left(f_{t_{1}}^{1}, \ldots, f_{t_{n}}^{n}\right) d \widehat{p}=\Phi\left(\int_{\Omega} f_{t_{1}}^{1} d \widehat{p}, \ldots, \int_{\Omega} f_{t_{n}}^{n} d \widehat{p}\right) .
$$

Proof. By Theorem 5 in [14] and the symmetric roles of $T$ and $\Omega$, we know that the exchangeability of $f_{\omega}^{i}$ implies the almost sure pairwise independence, hence uncorrelatedness (by the above remark), of $f_{t}^{i}, t \in T$. The result then follows from Theorem 5.6.

There are many variations of results concerning multilinear forms, orthogonality and uncorrelatedness. When multiple $t$ 's and $\omega$ 's are involved, different sub- $\sigma$ algebras of the product Loeb space appear. We give the following two relevant theorems to end this section.

Theorem 5.11. Suppose $f^{i}: T \times \Omega \rightarrow \mathbb{H}_{i}$ in $L^{n}\left(\widehat{\nu \otimes p}, \mathbb{H}_{i}\right)$ is almost surely orthogonal, $i=1,2, \ldots, n$ with $n \geq 2$. Let $\Psi: \mathbb{H}_{1} \times \cdots \times \mathbb{H}_{n} \rightarrow \mathbb{R}$ be a multilinear form. Then

(1) $E\left[\Psi\left(f^{1}\left(t_{1}, \omega\right), \ldots, f^{n}\left(t_{n}, \omega\right)\right) \mid L_{\nu^{n}}\left(\mathcal{A}^{n}\right) \otimes L_{p}(\mathcal{B})\right]=0$; 
(2) $E\left[\Psi\left(f^{1}\left(t, \omega_{1}\right), \ldots, f^{n}\left(t, \omega_{n}\right)\right) \mid L_{\nu}(\mathcal{A}) \otimes L_{p^{n}}\left(\mathcal{B}^{n}\right)\right]=0$;

(3) if $0 \leq k \neq l \leq n$ and $1 \leq k+l<2 n$, then

$$
E\left[\Psi\left(f^{1}\left(t_{1}, \omega_{1}\right), \ldots, f^{n}\left(t_{n}, \omega_{n}\right)\right) \mid \mathcal{U}(k, l ; n-k, n-l)\right]=0,
$$

where $\mathcal{U}(k, l ; n-k, n-l)$ denotes

$$
L_{\nu^{k} \otimes p^{l}}\left(\mathcal{A}^{k} \otimes \mathcal{B}^{l}\right) \otimes L_{\nu^{n-k} \otimes p^{n-l}}\left(\mathcal{A}^{n-k} \otimes \mathcal{B}^{n-l}\right) ;
$$

(4) for $\widehat{\nu^{n}}$-almost all $\left(t_{1}, \ldots, t_{n}\right) \in T^{n}$,

$$
\int_{\Omega} \Psi\left(f_{t_{1}}^{1}, \ldots, f_{t_{n}}^{n}\right) d \widehat{p}=0
$$

(5) for $\widehat{p^{n}}$-almost all $\left(\omega_{1}, \ldots, \omega_{n}\right) \in \Omega^{n}$,

$$
\int_{T} \Psi\left(f_{t}^{1}\left(\omega_{1}\right), \ldots, f_{t}^{n}\left(\omega_{n}\right)\right) d \widehat{\nu}(t)=0
$$

(6) if $0 \leq k \neq l \leq n$ and $1 \leq k+l<2 n$, then for any permutations $\sigma, \tau$ of $\{1,2, \ldots, n\}$ and for $\widehat{\nu^{k \otimes p}}$-almost all $\left(t_{\sigma_{1}}, \ldots, t_{\sigma_{k}}, \omega_{\tau_{1}}, \ldots, \omega_{\tau_{l}}\right)$ in $T^{k} \times \Omega^{l}$, the integral of the function $\Psi\left(f^{1}\left(t_{1}, \omega_{1}\right), \ldots, f^{n}\left(t_{n}, \omega_{n}\right)\right)$ with respect to the variables $\left(t_{\sigma_{k+1}}, \ldots, t_{\sigma_{n}}, \omega_{\tau_{l+1}}, \ldots, \omega_{\tau_{n}}\right)$ is zero. That is,

$$
\int_{T^{n-k} \times \Omega^{n-l}} \Psi\left(f_{t_{1}}^{1}\left(\omega_{1}\right), \ldots, f_{t_{n}}^{n}\left(\omega_{n}\right)\right) d \nu^{n-\widehat{k} \otimes p^{n-l}}\left(t_{\sigma_{i}}, \omega_{\tau_{j}}\right)_{i \geq k+1, j \geq l+1}=0 .
$$

Proof. By Theorem 4.3. (1) implies (4), (2) implies (5), and (3) implies (6). To prove (1), we start with any $A \in L_{\nu^{n}}(\mathcal{A} \otimes \cdots \otimes \mathcal{A}), B \in L_{p}(\mathcal{B})$, and set $A_{t_{2}, \ldots, t_{n}}=$ $\left\{t_{1} \in T,\left(t_{1}, t_{2}, \ldots, t_{n}\right) \in A\right\}$. Then

$$
\begin{aligned}
& \int_{A \times B} \Psi\left(f_{t_{1}}^{1}(\omega), \ldots, f_{t_{n}}^{n}(\omega)\right) d \widehat{\nu^{n} \otimes p}\left(t_{1}, \ldots t_{n}, \omega\right) \\
& =\int_{T^{n-1} \times B} \int_{A_{t_{2}, \ldots, t_{n}}} \Psi\left(f_{t_{1}}^{1}(\omega), \ldots, f_{t_{n}}^{n}(\omega)\right) d \widehat{\nu}\left(t_{1}\right) d \widehat{\nu^{n-1} \otimes} p\left(t_{2}, \ldots, t_{n}, \omega\right) \\
& =0
\end{aligned}
$$

because the almost sure orthogonality of $f^{1}$ implies that

$$
\begin{aligned}
& \int_{A_{t_{2}, \ldots, t_{n}}} \Psi\left(f^{1}\left(t_{1}, \omega\right), \ldots, f^{n}\left(t_{n}, \omega\right)\right) d \widehat{\nu}\left(t_{1}\right) \\
= & \Psi\left(\int_{A_{t_{2}, \ldots, t_{n}}} f^{1}\left(t_{1}, \omega\right) d \widehat{\nu}\left(t_{1}\right), \ldots, f^{n}\left(t_{n}, \omega\right)\right) \\
= & \Psi\left(0, \ldots, f^{n}\left(t_{n}, \omega\right)\right)=0
\end{aligned}
$$

by Lemmas 3.1 and 4.2. Thus we obtain (1) while (2) has a similar proof.

To prove (3) and (6), simply note that there is an index $i \in\{1, \ldots, n\}$ such that the pair $\left(t_{i}, \omega_{i}\right)$ is neither part of $\left(t_{\sigma(1)}, \ldots, t_{\sigma(k)}, \omega_{\tau(1)}, \ldots, \omega_{\tau(l)}\right)$ nor part of $\left(t_{\sigma(k+1)}, \ldots, t_{\sigma(n)}, \omega_{\tau(l+1)}, \ldots, \omega_{\tau(n)}\right)$ (i.e., $t_{i}$ belongs to one group while $\omega_{i}$ belongs to the other group). Then use a proof similar to the one above. 
We also have the following result for the uncorrelatedness.

Theorem 5.12. Suppose $f^{i}: T \times \Omega \rightarrow \mathbb{H}_{i} \in L^{n}\left(\widehat{\nu \otimes p}, \mathbb{H}_{i}\right)$ is almost surely uncorrelated, $i=1,2, \ldots, n$ with $n \geq 2$. Let $\Psi: \mathbb{H}_{1} \times \cdots \times \mathbb{H}_{n} \rightarrow \mathbb{R}$ be a multilinear form. For any fixed positive integer $m \leq n$, if $\tau$ is a map from $\{1, \ldots, n\}$ onto $\{1, \ldots, m\}$, then for $\nu^{n \otimes p^{m}}$-almost all $\left(t_{1}, \ldots, t_{n}, \omega_{1}, \ldots, \omega_{m}\right)$,

$$
\begin{aligned}
& E\left[\Psi\left(f^{1}\left(t_{1}, \omega_{\tau(1)}\right), \ldots, f^{n}\left(t_{n}, \omega_{\tau(n)}\right)\right) \mid L_{\nu^{n}}\left(\mathcal{A}^{n}\right) \otimes L_{p^{m}}\left(\mathcal{B}^{m}\right)\right] \\
= & \Psi\left(E f_{t_{1}}^{1}, \ldots, E f_{t_{n}}^{n}\right) .
\end{aligned}
$$

Proof. For any $A$ in $L_{\nu^{n}}\left(\mathcal{A}^{n}\right)$ and any $B$ in $L_{p^{m}}\left(\mathcal{B}^{m}\right)$, set $A_{t_{2}, \cdots, t_{n}}=\left\{t_{1} \in T\right.$ : $\left.\left(t_{1}, t_{2}, \ldots, t_{n}\right) \in A\right\}$. Then by Keisler's Fubini theorem and Lemma 3.1,

$$
\begin{aligned}
& \int_{A \times B} \Psi\left(f^{1}\left(t_{1}, \omega_{\tau(1)}\right), \ldots, f^{n}\left(t_{n}, \omega_{\tau(n)}\right)\right) d \nu^{n \otimes p^{m}} \\
= & \int_{T^{n-1} \times B} \int_{A_{t_{2}, \ldots, t_{n}}} \Psi\left(f^{1}\left(t_{1}, \omega_{\tau(1)}\right), \ldots, f^{n}\left(t_{n}, \omega_{\tau(n)}\right)\right) d \widehat{\nu}\left(t_{1}\right) d \nu^{n-1 \otimes} p^{m} \\
= & \int_{T^{n-1} \times B} \Psi\left(\int_{A_{t_{2}, \ldots, t_{n}}} f^{1}\left(t_{1}, \omega_{\tau(1)}\right) d \widehat{\nu}\left(t_{1}\right), \ldots, f^{n}\left(t_{n}, \omega_{\tau(n)}\right)\right) d \nu^{n-1 \otimes} p^{m} \\
= & \int_{T^{n-1} \times B} \Psi\left(\int_{A_{t_{2}, \ldots, t_{n}}} \mathrm{E} f_{t_{1}}^{1} d \widehat{\nu}\left(t_{1}\right), \ldots, f^{n}\left(t_{n}, \omega_{\tau(n)}\right)\right) d \nu^{n-1 \otimes} p^{m} \\
= & \int_{T^{n-1} \times B} \int_{A_{t_{2}, \ldots, t_{n}}} \Psi\left(\mathrm{E} f_{t_{1}}^{1}, \ldots, f^{n}\left(t_{n}, \omega_{\tau(n)}\right)\right) d \widehat{\nu}\left(t_{1}\right) d \nu^{n-1 \otimes} p^{m} \\
= & \int_{A \times B} \Psi\left(\mathrm{E} f_{t_{1}}^{1}, \ldots, f^{n}\left(t_{n}, \omega_{\tau(n)}\right)\right) d \nu^{n \otimes p^{m}}\left(t_{1}, \ldots, t_{n}, \omega_{1}, \ldots, \omega_{m}\right) \\
= & \ldots \ldots \\
= & \int_{A \times B} \Psi\left(\mathrm{E} f_{t_{1}}^{1}, \ldots, \mathrm{E} f_{t_{n}}^{n}\right) d \nu^{\nu^{n} \otimes p^{m}}\left(t_{1}, \ldots, t_{n}, \omega_{1}, \ldots, \omega_{m}\right),
\end{aligned}
$$

where $d \nu^{n-1 \otimes} p^{m}$ is shorthand for $d \nu^{n-1 \otimes} p^{m}\left(t_{2}, \ldots, t_{n}, \omega_{1}, \ldots, \omega_{m}\right)$. This completes the proof.

\section{Biorthogonal REPRESENTATion}

In this section we show that every $f \in L^{2}(\widehat{\nu \otimes p}, \mathbb{H})$ can be represented as the sum of a series of biorthogonal products and an almost surely orthogonal process as in Theorem 4.2 of [13].

Let $f \in L^{2}(\widehat{\nu \otimes p}, \mathbb{H})$. Define $K: L^{2}(\widehat{p}) \rightarrow L^{2}(\widehat{p})$ as follows. For $\varphi \in L^{2}(\widehat{p})$,

$$
K \varphi(\omega)=\int_{T \times \Omega}\left\langle f(t, \omega), f\left(t, \omega^{\prime}\right)\right\rangle \varphi\left(\omega^{\prime}\right) d \widehat{\nu \otimes p}\left(t, \omega^{\prime}\right), \omega \in \Omega .
$$

Then we have the following result.

Lemma 6.1. Suppose that $f \in L^{2}(\widehat{\nu \otimes p}, \mathbb{H})$ and the operator $K$ is defined by equation (1). Then $K$ is a self-adjoint, positive semi-definite, compact operator.

Proof. The routine proof, using in part well-known facts about Hilbert-Schmidt operators, is left to the reader. 
Let $\gamma_{1}, \gamma_{2}, \ldots$ be the non-increasing sequence of all the positive eigenvalues of $K$ with each eigenvalue being repeated up to its multiplicity. Let $\varphi_{1}, \varphi_{2}, \ldots$ be the corresponding eigenfunctions adjusted to form an orthonormal family. Set

$$
\psi_{n}(t)=\gamma_{n}^{-1 / 2} \int_{\Omega} \varphi_{n}(\omega) f(t, \omega) d \widehat{p}(\omega), n=1,2, \ldots
$$

Now we turn to the main result of this section. Recall that $\mathcal{U}=L_{\nu}(\mathcal{A}) \otimes L_{p}(\mathcal{B})$.

Theorem 6.2. For $f: T \times \Omega \rightarrow \mathbb{H}$ in $L^{2}(\widehat{\nu \otimes p}, \mathbb{H})$, the operator $K$ is defined by equation (1), with $\gamma_{1}, \gamma_{2}, \ldots$ eigenvalues of $K$ in non-increasing order repeated up to its multiplicity, $\varphi_{1}, \varphi_{2}, \ldots$ the corresponding eigenfunctions, forming a orthonormal system. Let $\psi_{1}, \psi_{2}, \ldots$ be defined by (2). Then $\psi_{1}, \psi_{2}, \ldots$ form an orthonormal system; and

$$
E(f \mid \mathcal{U})(t, \omega)=\sum_{n=1}^{\infty} \gamma_{n}^{1 / 2} \varphi_{n}(\omega) \psi_{n}(t)
$$

Moreover, $f$ can be decomposed as

$$
f=\sum_{n=1}^{\infty} \gamma_{n}^{1 / 2} \varphi_{n}(\omega) \psi_{n}(t)+\varepsilon
$$

where $\varepsilon=f-E(f \mid \mathcal{U})$ is an almost surely orthogonal vector-valued process.

Proof. We first demonstrate that $\psi_{1}, \psi_{2}, \ldots$ form an orthonormal system in $L^{2}(\widehat{p}, \mathbb{H})$. For any $m, n$, Lemma 3.1 and Theorem 3.2 imply that

$$
\begin{aligned}
& \left\langle\psi_{m}, \psi_{n}\right\rangle_{L^{2}(\widehat{\nu}, \mathbb{H})} \\
= & \gamma_{m}^{-1 / 2} \gamma_{n}^{-1 / 2} \int_{T}\left[\left\langle\int_{\Omega} \varphi_{m}(\omega) f_{t}(\omega) d \widehat{p}(\omega), \int_{\Omega} \varphi_{n}\left(\omega^{\prime}\right) f_{t}\left(\omega^{\prime}\right) d \widehat{p}\left(\omega^{\prime}\right)\right\rangle\right] d \widehat{\nu}(t) \\
= & \gamma_{m}^{-1 / 2} \gamma_{n}^{-1 / 2} \int_{T}\left[\int_{\Omega}\left\langle\varphi_{m}(\omega) f_{t}(\omega), \int_{\Omega} \varphi_{n}\left(\omega^{\prime}\right) f_{t}\left(\omega^{\prime}\right) d \widehat{p}\left(\omega^{\prime}\right)\right\rangle d \widehat{p}(\omega)\right] d \widehat{\nu}(t) \\
= & \gamma_{m}^{-1 / 2} \gamma_{n}^{-1 / 2} \int_{T}\left[\int_{\Omega} \int_{\Omega}\left\langle\varphi_{m}(\omega) f_{t}(\omega), \varphi_{n}\left(\omega^{\prime}\right) f_{t}\left(\omega^{\prime}\right)\right\rangle d \widehat{p}(\omega) d \widehat{p}\left(\omega^{\prime}\right)\right] d \widehat{\nu}(t) \\
= & \gamma_{m}^{-1 / 2} \gamma_{n}^{-1 / 2} \int_{T \times \Omega^{2}} \varphi_{m}(\omega) \varphi_{n}\left(\omega^{\prime}\right)\left\langle f_{t}(\omega), f_{t}\left(\omega^{\prime}\right)\right\rangle d \nu \otimes p^{2}\left(t, \omega, \omega^{\prime}\right) \\
= & \gamma_{m}^{-1 / 2} \gamma_{n}^{-1 / 2} \int_{\Omega} \varphi_{m}(\omega) K \varphi_{n}(\omega) d \widehat{p}(\omega) \\
= & \gamma_{m}^{-1 / 2} \gamma_{n}^{-1 / 2} \int_{\Omega} \varphi_{m}(\omega) \gamma_{n} \varphi_{n}(\omega) d \widehat{p}(\omega),
\end{aligned}
$$

which is zero if $m \neq n$, and one if $m=n$. Thus, $\psi_{1}, \psi_{2}, \ldots$ form an orthonormal system.

To prove (3), we need only prove that for all $A \in L_{\nu}(\mathcal{A}), B \in L_{p}(\mathcal{B})$, the integrals of the two sides of (3) with respect to $\widehat{\nu \otimes p}$ on $A \times B$ are equal. Let $\chi_{A}$ and $\chi_{B}$ be the indicator functions of $A$ and $B$. Note that by the self-adjointness of $K, \varphi \in \operatorname{ker}(K)$ iff $\varphi \perp \operatorname{range}(K)$. By Theorem 3.2 we can obtain

$$
\begin{aligned}
& \int_{A \times B} \mathrm{E}[f \mid \mathcal{U}] d \widehat{\nu \otimes p}=\int_{A \times B} f d \widehat{\nu \otimes p}=\int_{T \times \Omega} \chi_{A} \chi_{B} f d \widehat{\nu \otimes p} \\
= & \int_{T \times \Omega} \chi_{A} \pi_{\text {rangeK }}\left(\chi_{B}\right) f d \widehat{\nu \otimes p}+\int_{T \times \Omega} \chi_{A} \pi_{k e r K}\left(\chi_{B}\right) f d \widehat{\nu \otimes p},
\end{aligned}
$$


where $\pi_{\text {range } K}$ and $\pi_{\text {ker } K}$ are the orthogonal projections from $L^{2}(\widehat{p}, \mathbb{H})$ to range $K$ (range of $K$ ) and $\operatorname{ker} K$ (kernel of $K$ ), respectively. By equation (2), we see that

$$
\begin{aligned}
& \int_{T \times \Omega} \chi_{A} \pi_{\text {rangeK }}\left(\chi_{B}\right) f d \widehat{\nu \otimes p} \\
= & \int_{T \times \Omega} \chi_{A}(t) \sum_{n=1}^{\infty} \int_{\Omega} \chi_{B}\left(\omega^{\prime}\right) \varphi_{n}\left(\omega^{\prime}\right) d \widehat{p}\left(\omega^{\prime}\right) \varphi_{n}(\omega) f(t, \omega) d \widehat{\nu}(t) d \widehat{p}(\omega) \\
= & \int_{T \times \Omega} \chi_{A}(t) \chi_{B}\left(\omega^{\prime}\right) \sum_{n=1}^{\infty} \varphi_{n}\left(\omega^{\prime}\right) \gamma_{n}^{1 / 2} \psi_{n}(t) d \widehat{\nu}(t) d \widehat{p}\left(\omega^{\prime}\right) \\
= & \int_{A \times B} \sum_{n=1}^{\infty} \gamma_{n}^{1 / 2} \varphi_{n}(\omega) \psi_{n}(t) d \widehat{\nu \otimes p}(t, \omega) .
\end{aligned}
$$

Therefore, we need only show that

$$
\int_{T \times \Omega} \chi_{A} \pi_{k e r K}\left(\chi_{B}\right) f d \widehat{\nu \otimes p}=0 .
$$

By Theorem 3.2 ,

$$
\int_{T \times \Omega} \chi_{A} \pi_{k e r K}\left(\chi_{B}\right) f d \widehat{\nu \otimes p}=\int_{T} \chi_{A} \int_{\Omega} \pi_{k e r K}\left(\chi_{B}\right) f d \widehat{p} d \widehat{\nu} .
$$

So we obtain the desired result if we show $\int_{\Omega} \pi_{\text {kerK }}\left(\chi_{B}\right) f_{t} d \widehat{p}=0$ for $\widehat{\nu}$-almost all $t \in T$. To this end, we show that for any $\varphi \in \operatorname{ker} K, \int_{\Omega} \varphi(\omega) f(t, \omega) d \widehat{p}(\omega)=0$ for $\widehat{\nu}$-almost all $t$. In fact,

$$
\begin{aligned}
& \int_{T}\left\|\int_{\Omega} \varphi f d \widehat{p}\right\|^{2} d \widehat{\nu} \\
= & \int_{T}\left\langle\int_{\Omega} \varphi(\omega) f(t, \omega) d \widehat{p}(\omega), \int_{\Omega} \varphi\left(\omega^{\prime}\right) f\left(t, \omega^{\prime}\right) d \widehat{p}\left(\omega^{\prime}\right)\right\rangle d \widehat{\nu}(t) \\
= & \int_{T} \int_{\Omega} \varphi(\omega)\left\langle f(t, \omega), \int_{\Omega} \varphi\left(\omega^{\prime}\right) f\left(t, \omega^{\prime}\right) d \widehat{p}\left(\omega^{\prime}\right)\right\rangle d \widehat{p}(\omega) d \widehat{\nu}(t) \\
= & \int_{T} \int_{\Omega} \int_{\Omega} \varphi(\omega) \varphi\left(\omega^{\prime}\right)\left\langle f(t, \omega), f\left(t, \omega^{\prime}\right)\right\rangle d \widehat{p}\left(\omega^{\prime}\right) d \widehat{p}(\omega) d \widehat{\nu}(t) \\
= & \int_{\Omega} \varphi(\omega) K \varphi(\omega) d \widehat{p}(\omega)=0 .
\end{aligned}
$$

Note that (4) follows from (3) and Theorem 4.3.

Remark 6.3. Define $\widetilde{K}: L^{2}(\widehat{\nu}, \mathbb{H}) \rightarrow L^{2}(\widehat{\nu}, \mathbb{H})$ such that for $\psi \in L^{2}(\widehat{\nu}, \mathbb{H})$,

$$
\widetilde{K} \psi(t)=\int_{T} \int_{\Omega}\left\langle f\left(t_{1}, \omega\right), \psi\left(t_{1}\right)\right\rangle f(t, \omega) d \widehat{p}(\omega) d \widehat{\nu}\left(t_{1}\right), t \in T .
$$

Then it is easy to verify that $\widetilde{K}$ is a compact operator, and for each $n, \psi_{n}$ is an eigenfunction of $\widetilde{K}$ with corresponding eigenvalue $\gamma_{n}$. We further remark that it is also true that $\varphi_{n}(\omega)=\gamma_{n}^{-1 / 2} \int_{T}\left\langle f_{\omega}(t), \psi_{n}(t)\right\rangle \widehat{\nu}(t)$ for $n=1,2, \ldots$

We conclude this section by mentioning that the biorthogonality representation can also be of the above form but with $\varphi_{n}$ belonging to $L^{2}(\widehat{p}, \mathbb{H})$ and $\psi_{n}$ belonging to $L^{2}(\widehat{\nu})$ for all $n$. 


\section{REFERENCES}

[1] S. Albeverio, J. E. Fenstad, R. Høegh Krohn, T. Lindstrøm, Nonstandard Methods in Stochastic Analysis and Mathematical Physics, Academic Press, Orlando, Florida, 1986. MR 88f:03061

[2] R. M. Anderson, A nonstandard representation of Brownian motion and Itô integration, Israel J. Math. 25(1976), 15-46. MR 57:4311

[3] J. Berger, H. Osswald, Y. N. Sun, J. L. Wu, On nonstandard product measure spaces, Illinois J. Math. 46(2002), 319-330.

[4] V. S. Borkar, Probability Theory, an Advanced Course, Springer-Verlag, New York, 1995. MR 98e:60001

[5] G. Da Prato, and J. Zabczyk, Stochastic equations in infinite dimensions, Cambridge University Press, Cambridge, 1992. MR 95g:60073

[6] J. Diestel and J. J. Uhl, Jr., Vector Measures, American Mathematical Society, Providence, 1977. MR 56:12216

[7] H. J. Keisler, Hyperfinite model theory, in Logic Colloquium 76 (R. O. Gandy and J. M. E. Hyland, eds.) North-Holland, Amsterdam, 1977. MR 58:10421

[8] H. J. Keisler and Y. N. Sun, A metric on probabilities, and products of Loeb spaces, J. London Math. Soc., to appear.

[9] P. A. Loeb, Conversion from nonstandard to standard measure spaces and applications in probability theory, Trans. Amer. Math. Soc., 211(1975), 113-122. MR 52:10980

[10] P. A. Loeb, A functional approach to nonstandard measure theory, Contemporary Mathematics, Vol. 26(1984), 251-261. MR 86b:28026

[11] P. A. Loeb and M. Wolff, Nonstandard Analysis for the Working Mathematician, Kluwer Academic Publishers, Amsterdam, 2000. MR 2001e:03006

[12] H. Osswald, Malliavin calculus in abstract Wiener space using infinitesimals, Advances in Mathematics 176(2003), 1-37.

[13] Y. N. Sun, A theory of hyperfinite processes: the complete removal of individual uncertainty via exact LLN, Journal of Mathematical Economics 29(1998), 419-503. MR 99j:28020

[14] Y. N. Sun, The almost equivalence of pairwise and mutual independence and the duality with exchangeability, Probab. Theory and Relat. Fields 112(1998), 425-456. MR 2000m:60002

[15] K. Yosida, Functional Analysis, Springer-Verlag, Berlin, 1980. MR 82i:46002

Department of Mathematics, University of Illinois, 1409 West Green St., Urbana, ILLINOIS 61801

E-mail address: loeb@math.uiuc.edu

Mathematisches Institut der LMU-München, Theresienstr.39, D-80333 München, GerMANY

E-mail address: Horst.Osswald@mathematik.uni-muenchen.de

Institute for Mathematical Sciences, National University of Singapore, 3 Prince George's Park, Singapore 118402, Republic of Singapore, - And - Department of Mathematics, National University of Singapore, 2 Science Drive 2, Singapore 117543, Republic OF SINGAPORE

E-mail address: matsuny@nus.edu.sg

Department of Mathematics, National University of Singapore, 2 Science Drive 2 , Singapore 117543, Republic of Singapore, - And - School of Mathematical Sciences, Peking University, Beijing 100871, People's Republic of China

E-mail address: matzzx@nus.edu.sg 